Proceedings

\title{
New perspectives for the diagnosis and planning of urban mo- bility after COVID-19: the case study of Cartagena
}

Salvador García-Ayllón ${ }^{2}$

1 Technical University of Cartagena, Spain
* Correspondence: salvador.ayllon@upct.es

\begin{abstract}
Sustainable Urban Mobility Plans (SUMP) are increasingly popular planning tools in cities with environmental issues where numerous actions are usually proposed to reduce pollution from urban transport. The impact of the pandemic caused by COVID-19 on urban pollution in our cities is a proven fact, although its mechanisms are not known in great detail. The change in urban mobility patterns due to the restrictions imposed on the population during lockdown is a phenomenon that can be parameterized and studied from the perspective of spatial analysis. This study proposes an analysis of the guiding parameters of these changes from the perspective of spatial analysis. To do so, the case study of the city of Cartagena, a medium-sized city in Spain, has been analyzed throughout the period of mobility restrictions due to COVID-19. By means of a geostatistical analysis, changes in urban mobility patterns and the modal distribution of transport have been correlated with the evolution of environmental air quality indicators in the city.
\end{abstract}

Keywords: urban mobility; COVID-19; Cartagena; spatial analysis; geostatistics.

\section{Introduction}

The pandemic caused by the SARS-CoV-2 virus has brought about a very profound New perspectives for the diagnosis and planning of urban mobility after COVID-19: the case study of Cartagena. SUPTM 2022 conference proceedings sciforum-051392.

https://doi.org/10.31428/10317/10607

Publisher's Note: UPCT and Sciforum stays neutral with regard to jurisdictional claims in published maps and institutional affiliations.

Copyright: (c) 2022 by the authors. Submitted for possible open access publication under the terms and conditions of the Creative Commons Attribution (CC BY) license (https://creativecommons.org/licens es/by/4.0/). change in our society's way of life at all levels. One of the aspects on which the pandemic has had a greater impact has been the field of urban mobility, due to the restrictions imposed in many countries. This has caused a temporary transformation of mobility patterns in cities, the impact of which is only partially known. At a general level, the first studies on the matter highlighted that, during the time of the greatest restrictions on mobility in cities, pollution levels fell by $50 \%$ in developed countries [1].

Nevertheless, these first figures are only part of the phenomenon, since the subsequent changes caused by the pandemic in the behavior patterns of urban mobility are not limited to the transitory impact of the initial reduction of polluting gases caused by people remaining at home due to lockdown. The capacity restrictions in public transport, the greater use of the private vehicles because of the psychological effect of the possibility of contagion or the change in the lifestyle habits of users throughout this past and present period have had effects that should also be analyzed from a broader perspective at the environmental level [2].

To address this, we have studied the urban mobility patterns in the city of Cartagena (southeast Spain) during the pandemic from the perspective of spatial statistical analysis. Urban mobility patterns reflecting behavioral motifs in the city before and during the pandemic have been contrasted by means of GIS spatial analysis indicators. These patterns have also been statistically correlated at the spatial level in the city using geostatistical analysis tools to infer the region-specific evolution of the different environmental impacts caused by the pandemic.

\section{Area of study}

The study area is located in Cartagena, a medium-sized city in the southeast of Spain. 
Cartagena is a touristic and university city (the municipality has a registered population of about 220,000 inhabitants plus a floating population of another 100,000 inhabitants linked to tourism), it has an important endowment of bike lanes, and new transport alternatives are developing in them, such as personal mobility vehicles, without being a particularly noteworthy figure within the set of bike lane users (representing this set of alternatives almost 35,000 trips per day). Pedestrian movements constitute a major component of mobility, representing 360,000 of the 700,000 total trips that are made in the city on a daily basis (Figure 1).

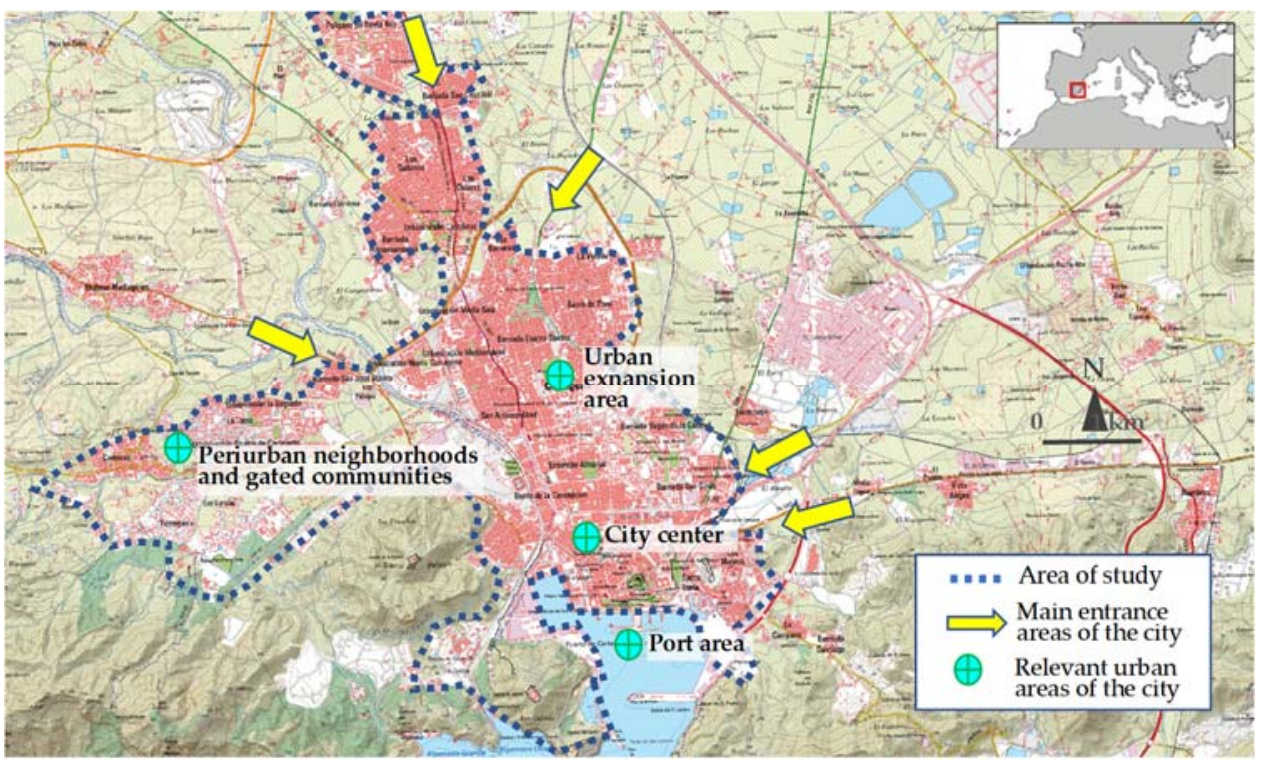

Figure 1. Delineation of the area of study (urban area of the city of Cartagena).

The city has several public transport alternatives such as urban and intercity buses (90,000 trips per day), medium and long-distance trains and a complete taxi service. Private transport coexists with some current transport alternatives based on the concept of collaborative economy such as car sharing or carpooling, with incipient commercial platforms which have a minor presence in the modal distribution of mobility in the city; this implies that private transport can be assumed to correspond to the traditional use of transport with a self-owned vehicle with almost 200,000 trips per day.

\section{Methodology}

For the purposes of this study, a spatial discretization of the urban area of the city has first been carried out. This process of compartmentalizing the urban continuum has been carried out based on the administrative structure of the city's neighborhoods (mainly taking into account the urban and social configuration of the city). The generation of specific sectors derived from urban configurations that require a singular treatment due to having specific characteristics (university areas, industrial estates, hospital spaces or large facilities, etc.) has then been added.

The data used in relation to public transport and the use of private vehicles have been obtained from the information provided by the concessionaires regarding public transport by bus, the associations of taxi drivers, and the traffic analyses periodically carried out by the City Council in several of its streets through the municipal traffic control center. As for the information on pedestrian mobility, by bicycle and through personal mobility vehicles, the data have been obtained through a series of surveys carried out during the development of the Sustainable Urban Mobility Plan of the city of Cartagena through apps with georeferenced information systems (see figure 2 left) that allowed to obtain spatial information on the itineraries used, the frequencies of use and the profiles of non-users(see figure 2 right). 

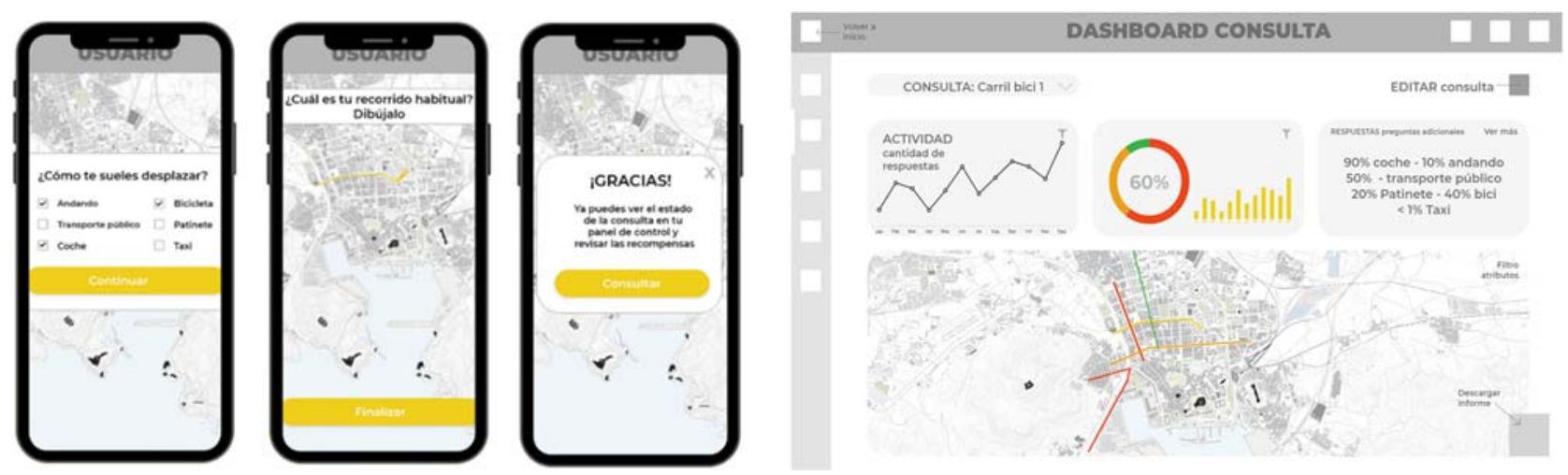

Figure 2. Apps with georeferenced information used for the development of surveys in Cartagena and example of survey software for spatial analysis of bike lane activity during the pandemic.

\section{Results and Discussion}

The results obtained based on the methodological framework described in the previous section are presented below in two phases. First, numerical values related to the change of urban mobility due to COVID-19 in the city of Cartagena are presented from an aggregate approach. Second, the way that urban mobility patterns and their environmental impact have evolved during the pandemic is shown in a segmented manner.

The pandemic has had a very significant effect at an overall level in the city of Cartagena in terms of urban mobility. Upon looking at the values of urban public transport by bus, we observe that the number of passengers decreased by one third in 2020, from 3.3 million to 2.2 million users in the city. However, that drop was not homogeneous over time; a much more pronounced decline in mobility can be noted initially, compared to a later recovery in mobility due to the easing of restrictions in subsequent months. Figures 3 and 4 present graphs showing average drops of $70 \%$ in demand in public transport use after the city went into lockdown in March and April 2020 due to the pandemic.

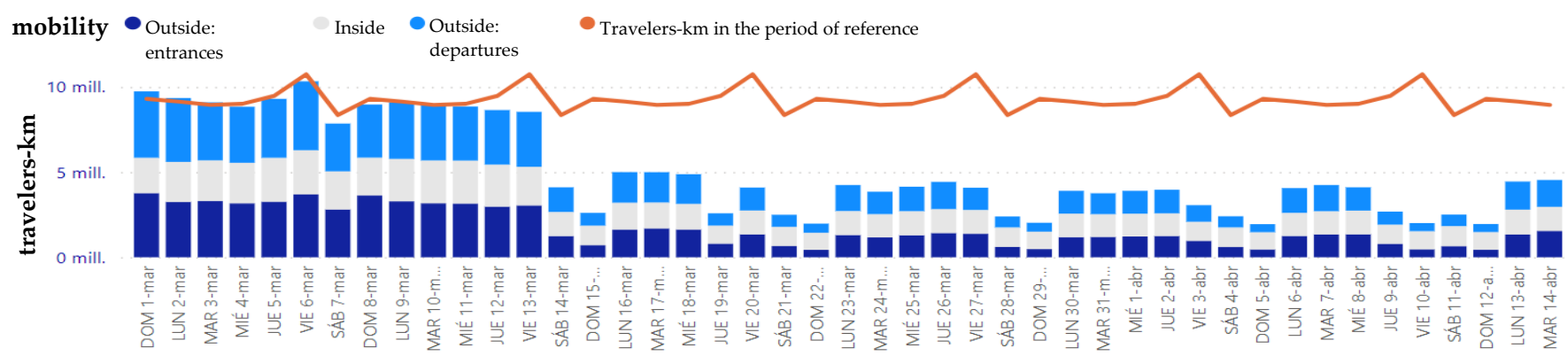

Figure 3. Evolution of daily mobility using public transport in Cartagena from the entry into force of mobility restrictions in 2020 (in blue) compared to the previous year 2019 (in red). Data source: Ministry of Transport of Spain and Cartagena City Council.

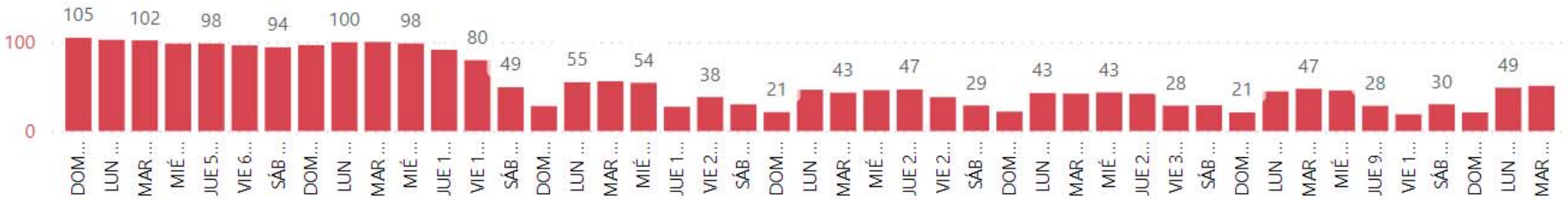

Figure 4. Percentage of daily mobility using public transport in Cartagena in March and April 2020 compared to the same days in 2019. Data source: Ministry of Transport of Spain and Cartagena City Council.

With the partial return to normality and the relaxation of mobility restrictions brought about by the appearance of vaccines, the figures for the reduction of trips and use of transport in the initial months of the pandemic have been progressively nuanced in the 
city of Cartagena. In graphs 5 and 6 we can see how up to $70-75 \%$ of the pre-pandemic 1 values had recovered globally in the months of March and April 2021.

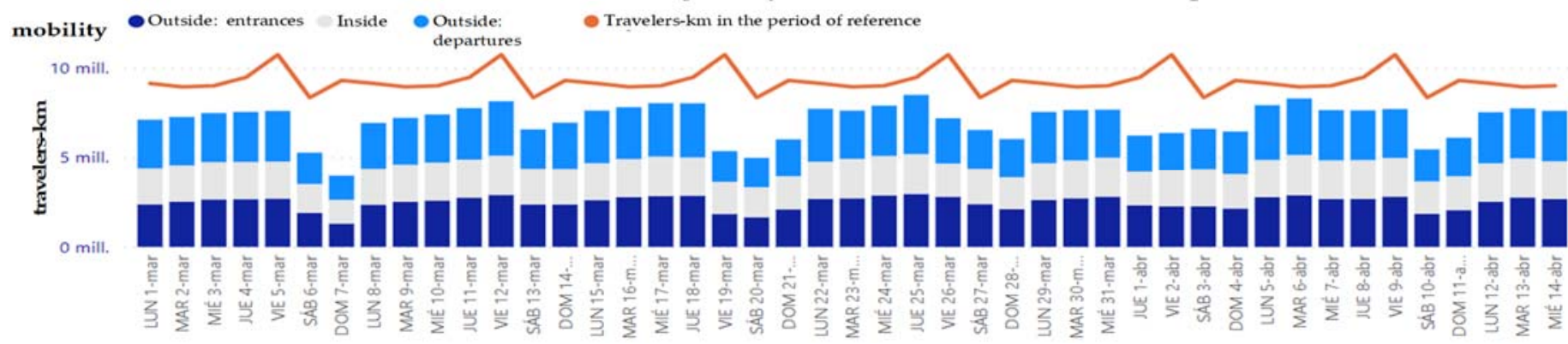

Figure 5. Evolution of daily mobility using public transport in Cartagena in March and April 2021 (in blue) compared to 2019 (in red). Data source: Ministry of Transport of Spain and Cartagena City Council.

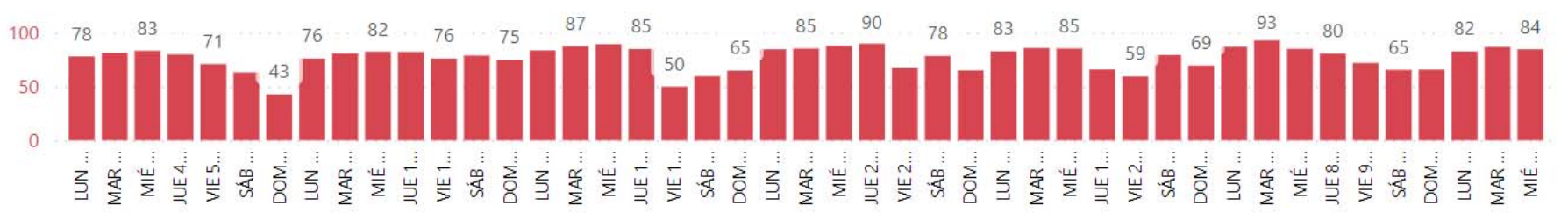

Figure 6. Percentage of daily mobility using public transport in Cartagena in March and April 2021 compared to the same days in 2019. Data source: Ministry of Transport of Spain and Cartagena City Council.

If we analyze these data in an aggregate way for each of the urban mobility alternatives, we can verify that there has been a substantial alteration in the usage patterns of urban mobility during the pandemic. The transfer flows of the modal split obtained through the app-based surveys, the data provided by the public transport concession companies and the monitoring data of the City Council's traffic control center have been represented in a summarized and schematic way through a Sankey diagram in Figure 7. 2019

2020

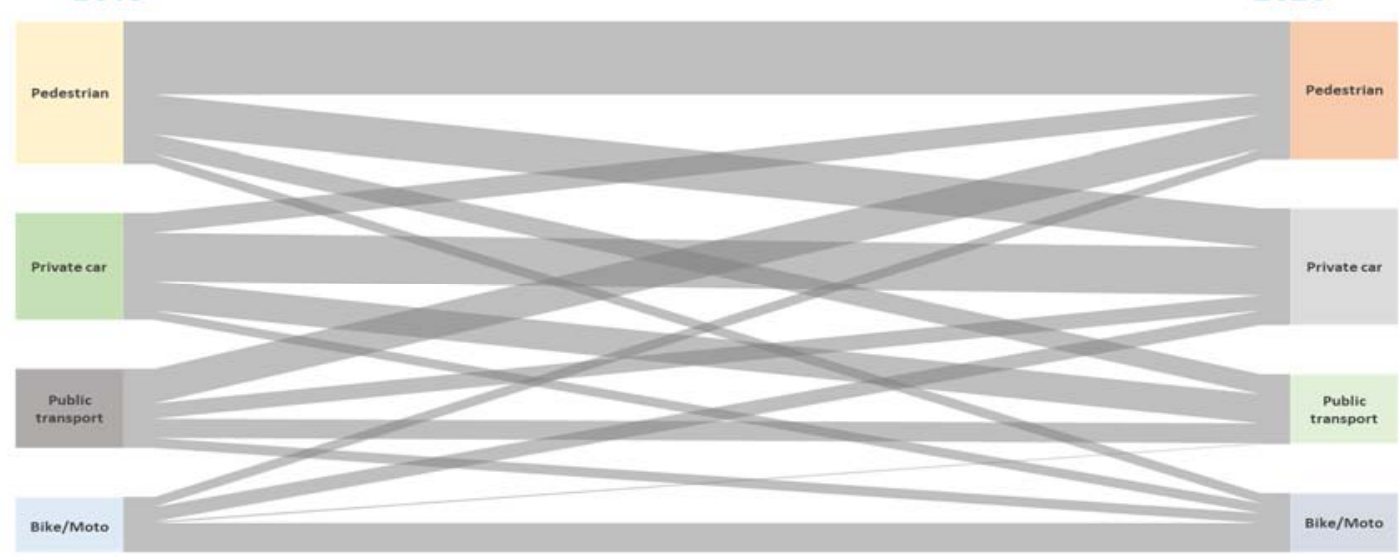

Figure 7. Simplified Sankey diagram of modal distribution transfer between 2019 and 2020 due to the pandemic.

Funding: This research received no external funding.

Conflicts of Interest: The authors declare no conflict of interest.

\section{References}

1. Garcia-Ayllon, S.; Hontoria, E.; Munier, N. The Contribution of MCDM to SUMP: The Case of Spanish Cities during 2006-2021. Int. J. Environ. Res. Public Heal. 2022, 19, 294.

2. García-Ayllón, S.; Kyriakidis, P. Spatial Analysis of Environmental Impacts Linked to Changes in Urban Mobility Patterns during COVID-19: Lessons Learned from the Cartagena Case Study. L. 2022, 11, 81. 